CORRIGENDUM

\title{
Cloud computing for genomic data analysis and collaboration
}

Ben Langmead \& Abhinav Nellore

Nature Reviews Genetics doi:10.1038/nrg.2017.113 (2018)

The above article originally stated "FireCloud and CGC rely on AWS and the Google Cloud Platform for computing and data storage. In addition to charges for these commercial services, users pay convenience surcharges." The second sentence was incorrect, as pointed out to and independently verified by the authors, and has been removed. Also, an incorrect citation was given for reference 66. The citation should have been: Bray, N. L., Pimentel, H., Melsted, P. \& Pachter, L. Near-optimal probabilistic RNA-seq quantification. Nat. Biotech. 34, 525-527 (2016). Finally, reference 67 referred to an older version of the CWL specification and has been updated. The article has been corrected online. The authors apologize for these errors. 